\title{
Arrival time distributions of very high energy cosmic ray muons in MACRO
}

\section{The MACRO Collaboration}

S.P. Ahlen ${ }^{a}$, M. Ambrosio ${ }^{b}$, G. Auriemma ${ }^{c, 1}$, A. Baldini ${ }^{d}$, G.C. Barbarino ${ }^{b}$,

B. Barish ${ }^{\text {e }}$, G. Battistoni ${ }^{\mathrm{f}}$, R. Bellotti ${ }^{\mathrm{g}}$, C. Bemporad ${ }^{\mathrm{d}}$, P. Bernardini ${ }^{\text {h, }}$, H. Bilokon ${ }^{\mathrm{f}}$, V. Bisi ${ }^{\mathrm{i}}$, C. Bloise ${ }^{\mathrm{f}}$, S. Bussino ${ }^{\mathrm{c}}$, F. Cafagna ${ }^{\mathrm{g}}$, M. Calicchio ${ }^{\mathrm{g}}$, P. Campana ${ }^{\mathrm{f}}$, S. Cecchini ${ }^{\mathrm{j}, 2}$, F. Cei ${ }^{\mathrm{d}}$, V. Chiarella ${ }^{\mathrm{f}}$, P. Chrysicopoulou ${ }^{\mathrm{c}}$, R. Cormack a, S. Coutu ${ }^{\text {e}}$, C. De Marzo ${ }^{g}$, G. De Cataldo ${ }^{g}$, M. De Vincenzi ${ }^{c}$, A. Di Credico ${ }^{\circ}$, E. Diehl ${ }^{\mathrm{m}}$, O. Erriquez ${ }^{\mathrm{g}}$, M. Fabbri ${ }^{\mathrm{j}}$, C. Favuzzi ${ }^{\mathrm{g}}$, C. Forti ${ }^{\mathrm{f}}$,

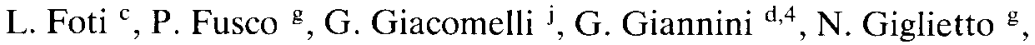
P. Giubellino ${ }^{\text {i }}$, M. Grassi ${ }^{\mathrm{d}}$, P. Green ${ }^{\mathrm{k}, 5}$, A. Grillo ${ }^{\mathrm{f}}$, F. Guarino ${ }^{\mathrm{b}}$, C. Gustavino ${ }^{\text {p }}$, R. Heinz ${ }^{\text {n }}$, J. Hong ${ }^{\text {e, E. Iarocci }}{ }^{\text {i, }}$, S. Klein ${ }^{\text {a }}$, E. Lamanna ${ }^{c}$, C. Lane ${ }^{\ell}$, D. Levin ${ }^{\mathrm{m}}$, P. Lipari ${ }^{\mathrm{c}}$, G. Liu ${ }^{\text {e }}$, R. Liu ${ }^{\mathrm{e}}$, M. Longo ${ }^{\mathrm{m}}$, G. Ludlam ${ }^{\text {a }}$, G. Mancarella ${ }^{\text {h}}$, G. Mandrioli ${ }^{j}$, A. Margiotta-Neri ${ }^{j}$, A. Marin ${ }^{\text {a }}$, A. Marini ${ }^{\text {f, }}$ D. Martello ${ }^{\text {h }}$, G. Martellotti ${ }^{\text {c }}$, A. Marzari-Chiesa ', M. Masera i,

P. Matteuzzi ', D. Michael `, L. Miller " , P. Monacelli ", M. Monteno i, S. Mufson ${ }^{\text {n }}$, J. Musser ${ }^{\mathrm{n}}$, G. Osteria ${ }^{\mathrm{b}}$, B. Pal ${ }^{\mathrm{j}}$, O. Palamara ${ }^{\mathrm{h}}$, V. Patera ${ }^{\mathrm{f}}$, L. Patrizii ${ }^{j}$, R. Pazzi ${ }^{d}$, C. Peck ${ }^{\text {e }}$, J. Petrakis ${ }^{n, 3}$, S. Petrera ${ }^{h}$, N. Pignatano ${ }^{\text {e, }}$ P. Pistilli ${ }^{\text {h }}$, F. Predieri ${ }^{j}$, L. Ramello ${ }^{i}$, J. Reynoldson ${ }^{\text {p }, ~ F . ~ R o n g a ~}{ }^{f}$, G. Rosa ${ }^{c}$, G.L. Sanzani ${ }^{j}$, L. Satta ${ }^{\text {f.7 }}$, E. Scapparone ${ }^{j}$, K. Scholberg e ${ }^{\text {, A. Sciubba }}{ }^{\text {c. } 6}$, P. Serra-Lugaresi ${ }^{\text {j }}$, M. Severi ${ }^{\text {c }}$, C. Smith ${ }^{\mathrm{m}}$, P. Spinelli ${ }^{g}$, M. Spinetti ${ }^{\text {f }}$, M. Spurio ${ }^{\text {j, J. Steele }}{ }^{\text {e }}$, R. Steinberg ${ }^{\text {, J.L. Stone }}{ }^{\text {a }, ~ L . R . ~ S u l a k ~}{ }^{\text {a }}$, A. Surdo ${ }^{\text {h }}$, G. Tarlè ${ }^{\mathrm{m}}, \mathrm{V}$. Togo ${ }^{\mathrm{j}}, \mathrm{V}$. Valente ${ }^{\mathrm{f}}, \mathrm{C}$. Walter ${ }^{\mathrm{e}}$ and R. Webb ${ }^{\mathrm{k}}$

${ }^{a}$ Physics Department of Boston University, Boston, MA 02215, USA

${ }^{\mathrm{b}}$ Dipartimento di Fisica dell Unicersità di Napoli and INFN, Napels 80125, Italy

${ }^{c}$ Dipartimento di Fisica dell'Unicersità di Roma and INFN, Rome 00185, Italy

' Dipartimento di Fisica dell'Unicersità di Pisa and INFN, Pisa 56010, Italy

' California Institute of Technology, Pasadena, CA 91125, USA

${ }^{\mathrm{I}}$ Laboratori Nazionali di Frascati dell INFN, Frascati (Rome) 00044, Italy

g Dipartimento di Fisica dell'Unicersità di Bari and INFN, Bari 70126, Italy

${ }^{\mathrm{h}}$ Dipartimento di Fisica dell'Unicersità di Lecce and INFN, Lecce 73100, Italy

1 Also at Università della Basilicata, Potenza 85100, Italy.

2 Also at Istituto TESRE/CNR, Bologna, Italy.

3 Present address: Bartol Research Institute, University of Delaware, Newark, DE, USA.

4 Also at Università di Trieste and INFN, Trieste 34100, Italy.

5 Present address: Div. 9321, Sandia National Laboratory, Albuquerque, NM 87185, USA.

6 Also at Dipartimento di Energetica, Università di Roma, Rome 00185, Italy.

7 Also at Università dell'Aquila, L'Aquila 67100, Italy. 


\footnotetext{
' Dipartimento di Fisica dell'Università di Torino and INFN, Turin 10125, Italy

${ }^{i}$ Dipartimento di Fisica dell'Università di Bologna and INFN, Bologna 40126, Italy

${ }^{k}$ Department of Physics of Texas A \& M University, College Station, TX 77843, USA

' Department of Physics, Drexel Unicersity, Philadelphia, PA 19104, USA

${ }^{m}$ Department of Physics of the Unicersity of Michigan, Ann Arbor, MI 48109, USA

${ }^{n}$ Dept. of Physics, Dept. of Astronomy, Indiana Unicersity, Bloomington, IN 47405, USA

- Dipartimento di Fisica dell'Università dell'Aquila and INFN, L'Aquila 67100, Italy

${ }^{\mathrm{P}}$ Laboratori Nazionali del Gran Sasso dell'INFN, Assergi (L'Aquila) 67010 Italy
}

\author{
Received 9 July 1991 \\ (Revised 25 October 1991) \\ Accepted for publication 15 November 1991
}

\begin{abstract}
We present a study of the correlations in the arrival times of about $10^{6}$ single and multiple muons detected by the first two MACRO supermodules. The time correlations, from milliseconds to several hundreds of seconds, have been analyzed in terms of the random distribution, with which they are consistent. An analysis of the arrival times (up to $180 \mathrm{~ns}$ ) of the muons from the same multimuon event is also given.
\end{abstract}

\title{
1. Introduction
}

It is generally assumed that galactic cosmic rays have a random arrival time distribution. But there may be mechanisms which introduce time modulations. For example, Weekes suggested that the arrival times of cosmic rays originating from a nearby pulsar could be modulated with a frequency equal to the rotation frequency of the pulsar [1]. Similar effects could arise in other types of sources of cosmic rays. For charged primary cosmic rays (protons, light and heavy nuclei) the magnetic fields between the source and the earth will reduce or eliminate such modulations, with the exception of the highest energy cosmic rays. Although the characteristic period of the pulsar might be completely masked (because particles with slightly different pitch angles will have different flight times), the pulsed injection could still lead to a generally anomalous distribution on short times scales. Also the injection of cosmic rays from supernova-like events could yield similar detectable effects.

A number of experiments have reported a non-random component in the arrival times of very high energy cosmic rays [2,3]. Bhat et al. [2], analyzing Cherenkov light pulses produced in the atmosphere by the showers generated by protons and other hadronic cosmic rays with $E_{\mathrm{p}}>100 \mathrm{TeV}$, reported a correlation at times $\leqslant 40 \mathrm{~s}$. Badino et al. [3], using an underground detector in the Mont Blanc tunnel at a depth of $5000 \mathrm{hg} / \mathrm{cm}^{2}$ of standard rock, reported a similar signal at times of $\sim 38 \mathrm{~s}$ for muons with energies larger than $E_{\mu} \simeq 380 \mathrm{GeV}$, originating from primaries with $E_{\mathrm{p}} \geqslant 50 \mathrm{TeV}$.

Other experiments have not found any signal [4-10]. 
In this paper we report a study of the time distributions of single and multiple muons detected by the streamer tube system of the first two MACRO supermodules and by the scintillators of the first supermodule. MACRO is a large-area underground detector located in the Gran Sasso National Laboratory, Assergi, Italy, at an average depth of 4000 m.w.e. [11]. Its primary aims are the search for rare particles and rare phenomena in the cosmic radiation. Because of its size (140

$\mathrm{m}^{2}$ per supermodule) it can study high-energy cosmic rays with large statistics. The mean energy of the muons reaching MACRO from above is $\left\langle E_{\mu}\right\rangle \sim 200 \mathrm{GeV}$; the corresponding minimum muon energy at the surface is $1.4 \mathrm{TeV}$; the primary cosmic ray has an energy $\geqslant 20 \mathrm{TeV}[12]$. For events with muon multiplicity $\geqslant 2$ the mean primary energy is $\geqslant 200 \mathrm{TeV}$.

About $10^{6}$ muon events were observed in a period of about one year. The arrival time distributions of consecutive muons from milliseconds to several hundred seconds have been measured and analysed in terms of the random distribution function, with which they are consistent. A study is also made of the arrival times, from $18 \mathrm{~ns}$ to $180 \mathrm{~ns}$, of the muons from the same multi-muon event. Delayed muons may arise from decays of massive, quasi-stable particles. Thus the physics motivations for this search are quite different from those of the previous two searches, which are mainly astrophysical. Nevertheless we include them here because this paper is mainly concerned with a general search of time correlations arising from any cause.

\section{Data and analysis}

We have used data from the first supermodule (SM) and also from the combination of the first and second supermodules. One supermodule has dimensions $12 \mathrm{~m} \times 12 \mathrm{~m} \times 4.8 \mathrm{~m}, S \Omega=800 \mathrm{~m}^{2} \mathrm{sr}$. It is made of two horizontal layers of liquid scintillation counters, ten horizontal layers of limited streamer tubes and one horizontal layer of track-etch detectors. Three of the vertical sides are closed by six layers of streamer tubes and one layer of scintillators [11].

In the first SM three muon triggers were based only on scintillation information and one muon trigger was based only on streamer tube information. In the second SM only the muon streamer tube trigger was operational.

The muon samples were selected from the data using our software reconstruction stream. In order to further clean the sample, a few loose additional requirements on the streamer tube informations were added. The selection criteria were [11]:

(i) The OR of the following conditions:

$\geqslant 4$ planes of horizontal wires

$\geqslant 3$ planes of the West, the East or the North face wires. 
TABLE 1

Running periods and number of single and multiple muon events for the muon samples from the limited streamer tube system. Multiplicities larger than 1 were computed on the basis of wire tracks only. Two supermodules were operational in the last two periods. See text for period definitions

\begin{tabular}{clrrr}
\hline Period & Muons & Total & After cuts & SM \\
\hline A & single & 240970 & 227880 & 1 \\
& multiple & 9610 & 9020 & 1 \\
B & single & 179940 & 175410 & 1 \\
& multiple & 7260 & 6910 & 1 \\
C & single & 125280 & 109420 & $1+2$ \\
& multiple & 7810 & 7110 & $1+2$ \\
D & single & 445230 & 407420 & $1+2$ \\
& multiple & 21290 & 19820 & $1+2$ \\
\hline
\end{tabular}

The subsequent requirement for finding a track is based on the alignment of at least four horizontal streamer tubes hits (wires or strips);

(ii) Rejection of events that have a large electromagnetic shower and/or noise in the streamer tubes.
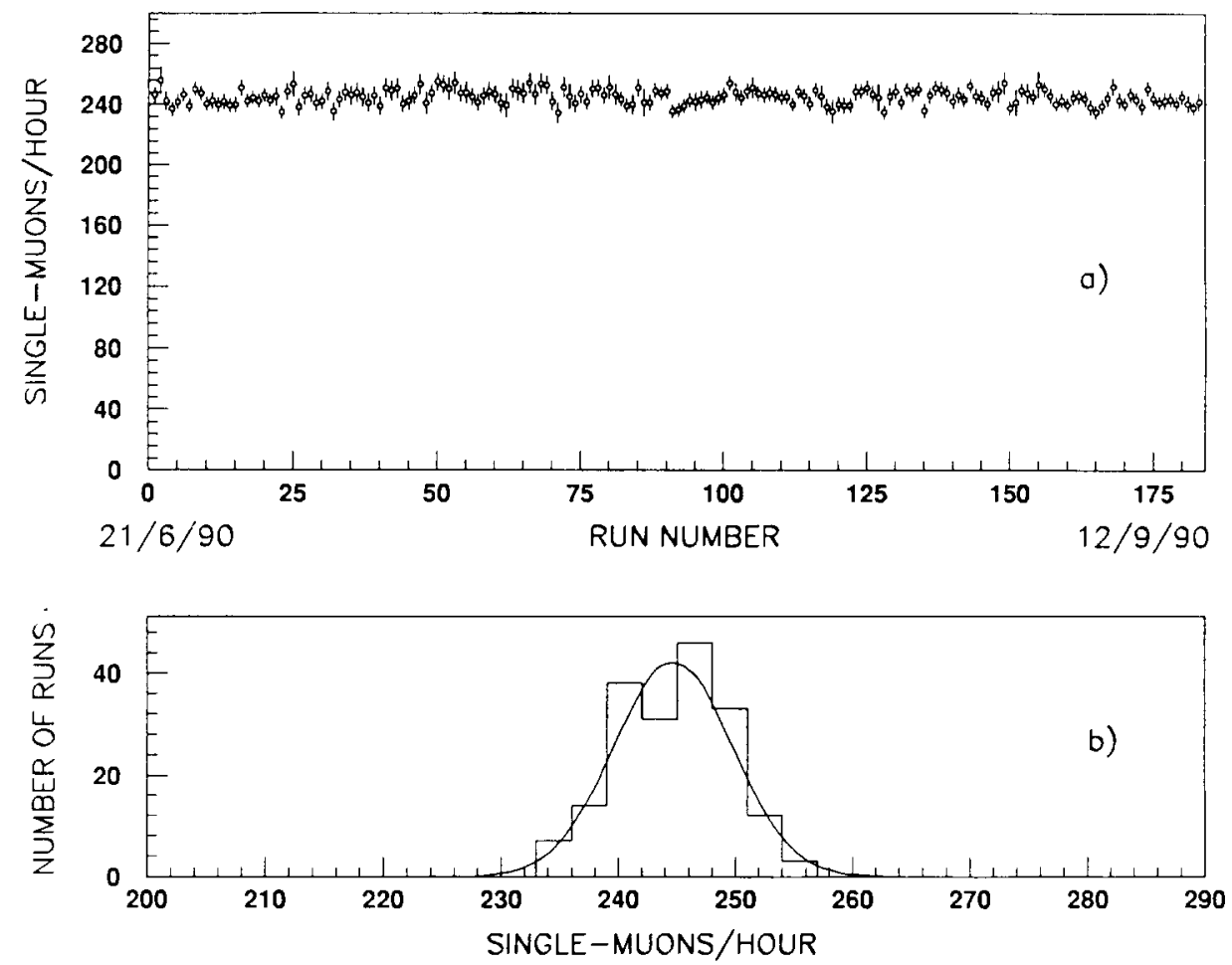

Fig. 1. The number of single muons per hour for the 183 selected runs of period (D). (a) Time evolution and (b) histogram; the solid curve is the fit with a gaussian distribution (its half width is consistent with what is expected on the basis of statistics). 
For all events selected in this way, a Data Summary Tape (mini-DST) was made, based on the event header; in particular, the time of arrival was obtained from the atomic clock, both for the streamer tube data and for scintillator data. The precision of the clock is about $1 \mathrm{~ms}$ absolute and $0.1 \mathrm{~ms}$ relative.

The data were divided into four periods (see table 1):

(A) From February 28 till May 30, 1989 (Spring 89);

(B) From November 11, 1989 till January 27, 1990 (Fall 89);

(C) From May 10 till June 15, 1990, two supermodules (Spring 90);

(D) From June 21 till September 12, 1990, two supermodules (Summer 90).

Events occurring during calibrations were rejected; runs which lasted less than 3 hours were not considered since they were test runs or ended because of power failures (a typical run lasted on the average 10 hours). A single muon event means a track with multiplicity equal to 1 both in the wire and strip streamer tube views. Multiple muons are events with multiplicity $>2$ in the wire view. In fig. 1 the number of single muon events per hour are plotted for period (D). The data falls well within the expected statistical fluctuations. Period (D) has larger statistics than the other periods and most of the detailed analysis is done on these data. In table 1 the number of events considered are also given. We have about an order of magnitude more data than previous experiments.

For the analysis of the time correlations we study for each muon arriving at time $t_{0}$, the time difference of the next muon $\left(t_{1}-t_{0}\right)$ and of the following four muons, $\left(t_{2}-t_{0}\right),\left(t_{3}-t_{0}\right),\left(t_{4}-t_{0}\right)$ and $\left(t_{5}-t_{0}\right)$.

The data were corrected for muon trigger dead times: after each trigger there is an average dead time of $70 \mathrm{~ms}$ in the first supermodule, where also scintillators are operational, $10 \mathrm{~ms}$ in the second supermodule where only streamer tubes are operational. In the periods with two supermodules $55 \%$ of the triggers were due to muons; the remaining triggers were due to non-muon events (natural radioactivity, etc). The effect of this dead time is apparent in the $\left(t_{1}-t_{0}\right)$ distribution at small times, see insert in fig. 2 . We estimated in period (D) a loss of 2670 muon events, out of 407420 . The $\left(t_{1}-t_{0}\right)$ distribution was fitted using data at times larger than $100 \mathrm{~ms}$, where no losses occur, and using data after repopulating the bins at small times: there is no change in the parameters. The $\left(t_{2}-t_{0}\right),\left(t_{3}-t_{0}\right),\left(t_{4}-t_{0}\right)$ and $\left(t_{5}-t_{0}\right)$ distributions were corrected by re-populating the DST with 2670 events at an average weighted time of $30 \mathrm{~ms}$ after every $407420 / 2670$ events.

The experimental time distributions, shown in figs. $2-5$, will be discussed in terms of the random distribution and of the Kolmogorov-Smirnov test.

\section{Fits of the data to the gamma function}

The time distribution data were fitted to the gamma function (poissonian of order $M$ )

$$
G(t ; \lambda, M)=N \frac{\lambda(\lambda t)^{M-1} \mathrm{e}^{-\lambda t}}{(M-1) !},
$$



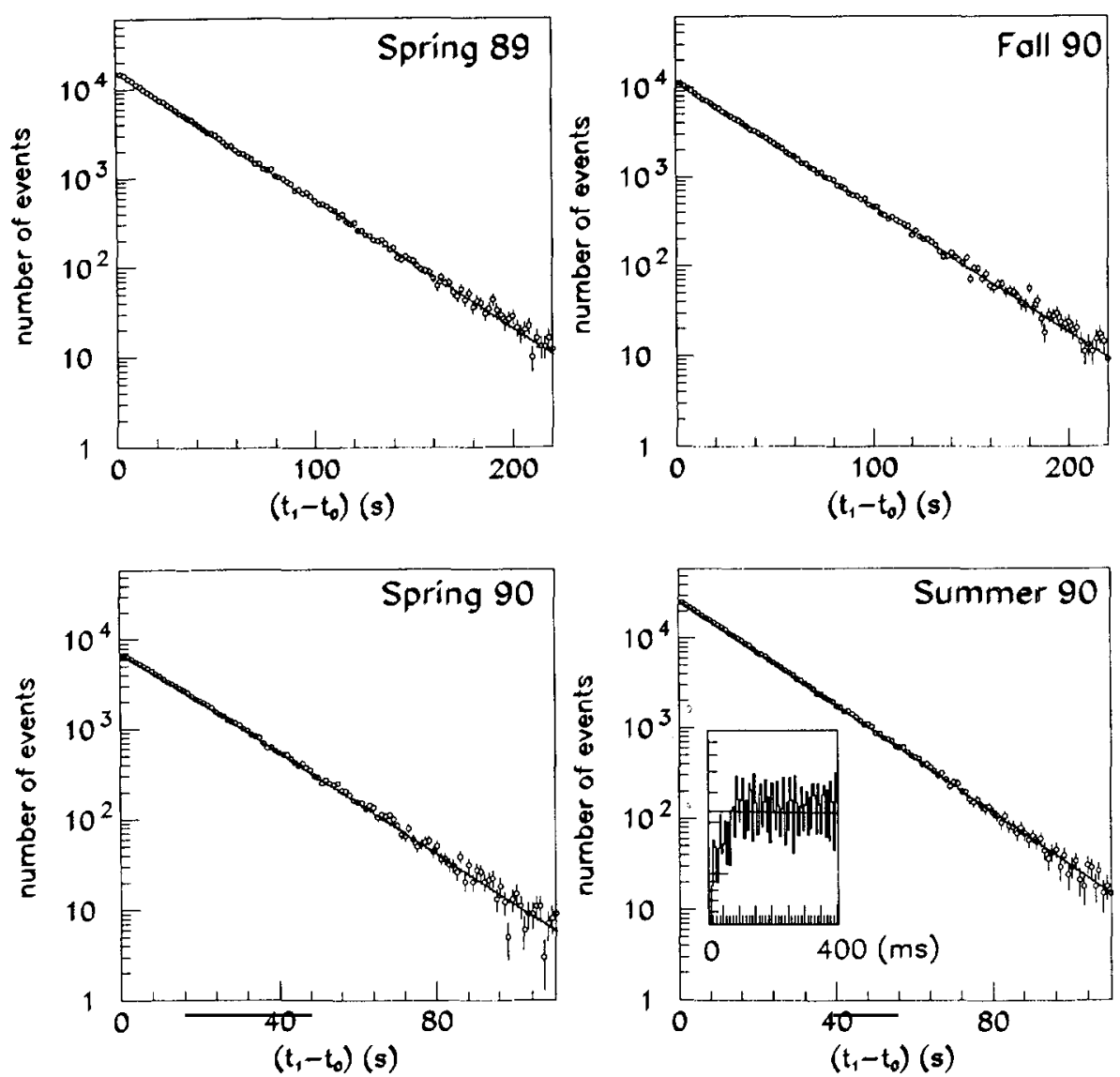

Fig. 2. Distributions of the time separation between two consecutive single muon events $\left(t_{1}-t_{0}\right)$ for the four periods (A)-(D) of table 1 . The lines (visible only at large times) represent the exponential fits of table 2 after correcting the data for dead time.

where $\lambda$ is the inverse of the mean value of the time difference $\left(t_{1}-t_{0}\right)$ between two consecutive muons, $M$ is the order of the distribution and $N$ is a normalization factor. For the $\left(t_{1}-t_{0}\right)$ fits we left $1 / \lambda$ as a free parameter, using $M=1$.

For $M=1$, eq. (1) reduces to an exponential function

$$
G(t ; \lambda, 1)=N \lambda \mathrm{e}^{-\lambda t} .
$$

The fits of the $\left(t_{1}-t_{0}\right)$ data to eq. (1) have reasonable $\chi^{2} /$ d.o.f. (typically equal to 1 , see table 2). The $\lambda$-coefficient should be numerically the same for all order distributions of the same period. In fact the experimental distributions of order $M$ $(M \geqslant 2)$ are well described by the gamma function of order $M$ with parameter $\lambda$ determined from the $\left(t_{1}-t_{0}\right)$ first-order distribution, but with $\chi^{2} /$ d.o.f. slightly larger than 1. Direct fits of the higher-order distributions to eq. (1) yield values of 

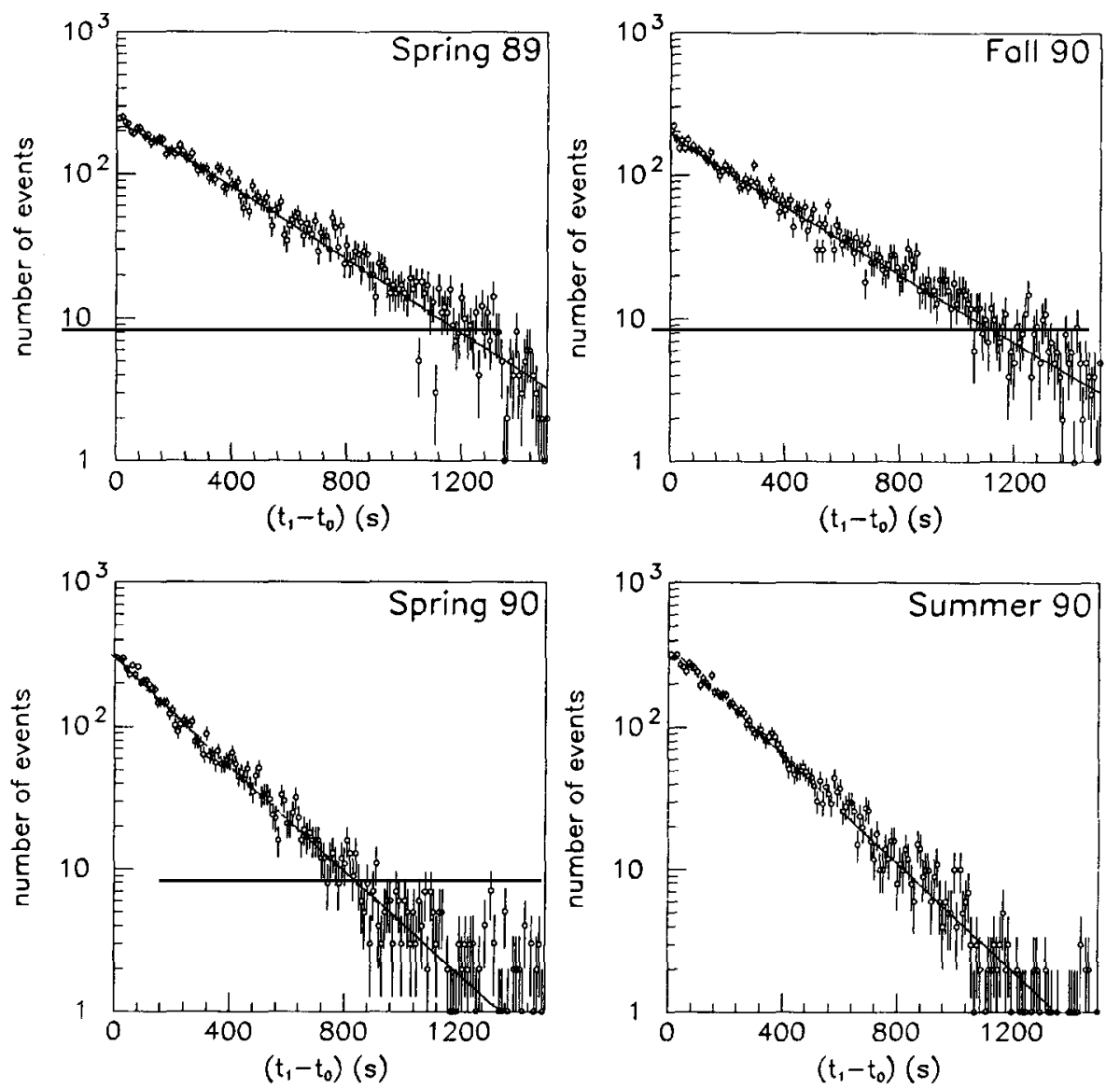

Fig. 3. The distributions of the time separation between two consecutive multi-muon events for samples (A)-(D). The lines represent the exponential fits of table 2.

$1 / \lambda$ equal, within errors, to that from the $\left(t_{1}-t_{0}\right)$ distribution yield $\chi^{2} /$ d.o.f. values of order 1 (see table 2 ).

\section{The Kolmogorov-Smirnov test}

In order to search for possible structures in the time arrival distributions, we have used the Kolmogorov-Smirnov test, which compares the cumulative distribution $F(x)$ of the experimental data with the expected random distribution $H(x)$. The measure of the deviation is $d=\max |F(x)-H(x)|$, where $F(x)$ and $H(x)$ are the cumulative distributions of $f(x)$ (data) and $h(x)$ (expected) respectively. In terms of this quantity, $F(x)$ agrees with $H(x, \lambda)$, where $\lambda$ is taken from the data, with a probability of compatibility between the expected and the measured 

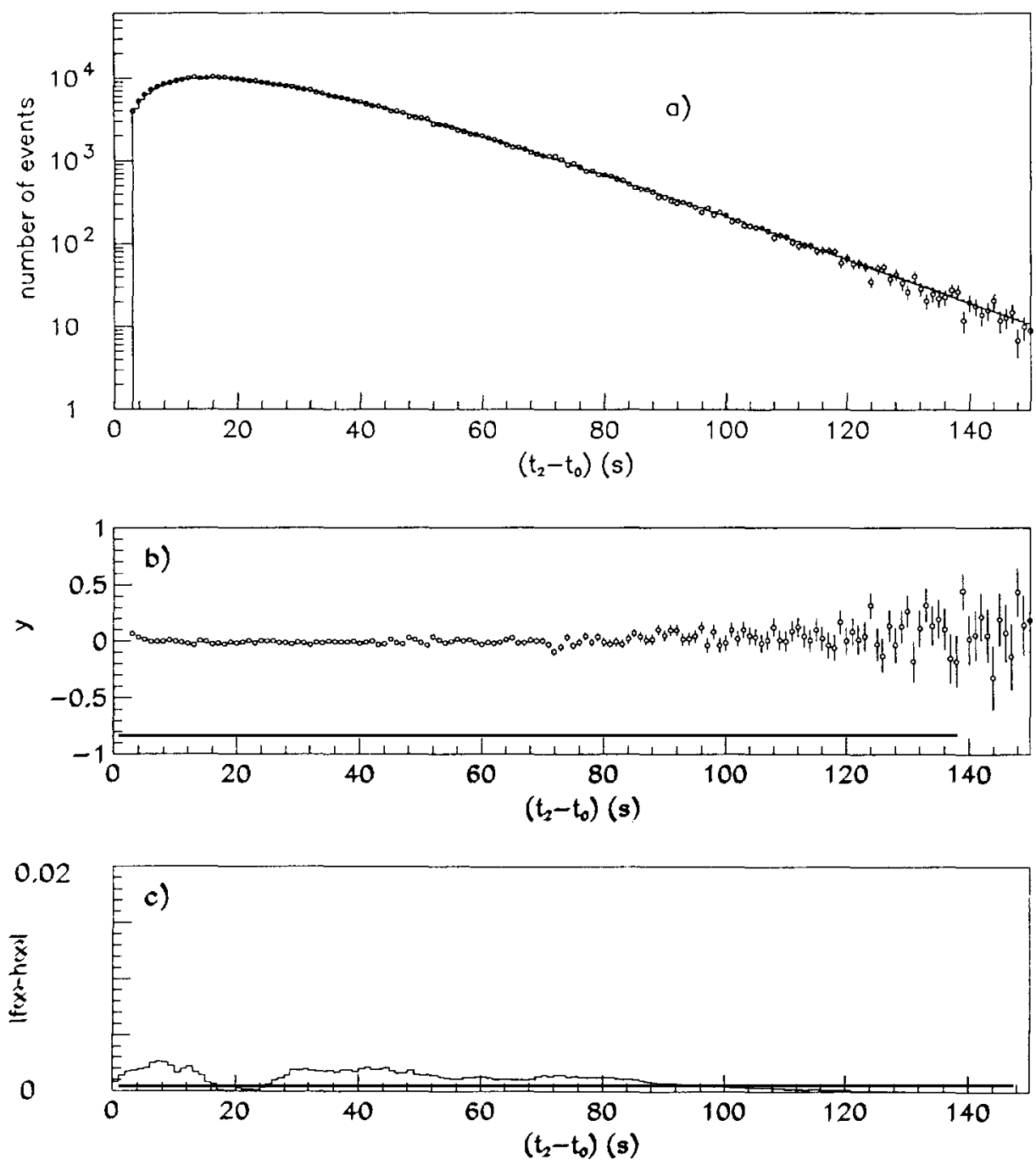

Fig. 4. (a) The $\left(t_{2}-t_{0}\right)$ time distribution for single muons of period (D). (b) The corresponding quantity $y$ is equal to the theoretical random $\left(t_{2}-t_{0}\right)$ distribution minus the experimental $\left(t_{2}-t_{0}\right)$ distribution divided by the theoretical random $\left(t_{2}-t_{0}\right)$ distribution vs. $\left(t_{2}-t_{0}\right)$. (c) The Kolmogorov-Smirnov test. The probability of the random distribution is $15.0 \%$.

distributions given by $P_{k}(d>$ observed $)=Q_{k s}(\sqrt{N} \mathrm{~d})$, where $Q_{k s}(x)=$ $2 \sum_{j=1}^{\infty}(-1)^{j-1} \mathrm{e}^{-j^{2} x^{2}}[13]$.

\section{Discussion of the time correlations with streamer tubes}

Fig. 2 shows the distributions of the time separation between consecutive single muon events for the four data periods mentioned above. The distributions are 



Fig. 5. Time distributions of $\left(t_{3}-t_{0}\right),\left(t_{4}-t_{0}\right)$ and $\left(t_{5}-t_{0}\right)$ for multiple muons of period (D). The lines are obtained from the fits of the $\left(t_{1}-t_{0}\right)$ data to the gamma function, see table 2.

clearly exponential, indicating the random nature of the bulk of the cosmic ray muon arrival times. Table 2 gives the parameter $\lambda$ of eq. (1) after fitting the distribution to the data. The fit has a reasonable $\chi^{2} /$ d.o.f. Also the results of the Kolmogorov-Smirnov tests is given in table 2. The slopes of the distributions for periods (C) and (D) are about twice those for period (A) and (B) because of the different acceptance.

Fig. 6 shows for the $\left(t_{1}-t_{0}\right)$ single muon data of period (D): (a) the percentage difference between the data and the fitted random distribution and (b) the result of the Kolmogorov-Smirnov test: $|F(x)-H(x)|$ versus $\left(t_{1}-t_{0}\right)$; the fit to the random distribution yields a probability of $88.4 \%$.

Fig. 3 shows the time distributions $\left(t_{1}-t_{0}\right)$ for multiple muon events of periods (A)-(D). The multimuon selection corresponds to selecting higher energy primaries. Also these distributions are well represented by exponential functions. 
TABLE 2

Results of the fits of the $M$-fold time distributions for single and multiple muon events to the gamma function [eq. (1)] for the data of period (D). Notice that the fits use data points in finer bins than what is shown in the figures

\begin{tabular}{lccc}
\hline Distribution & \multicolumn{2}{c}{ Fit to each distribution } & $\begin{array}{c}\text { Probability of } \\
\text { Kolmogorov test } \\
(\%)\end{array}$ \\
\cline { 2 - 3 } & $\begin{array}{c}1 / \lambda \\
(\mathrm{s})\end{array}$ & $\chi^{2} /$ d.o.f. & \\
\hline Single muons & & & 88.4 \\
$\left(t_{1}-t_{0}\right)$ & $14.66 \pm 0.03$ & $228 / 249$ & 13.8 \\
$\left(t_{2}-t_{0}\right)$ & $14.77 \pm 0.03$ & $325 / 346$ & 32.2 \\
$\left(t_{3}-t_{0}\right)$ & $14.73 \pm 0.02$ & $478 / 399$ & 49.6 \\
$\left(t_{4}-t_{0}\right)$ & $14.70 \pm 0.02$ & $495 / 449$ & 64.2 \\
$\left(t_{5}-t_{0}\right)$ & $14.69 \pm 0.02$ & $702 / 499$ & \\
Multiple muons & & & 97.8 \\
$\left(t_{1}-t_{0}\right)$ & $306 \pm 3$ & $194 / 199$ & 63.3 \\
$\left(t_{2}-t_{0}\right)$ & $306 \pm 2$ & $294 / 299$ & 86.2 \\
$\left(t_{3}-t_{0}\right)$ & $306 \pm 2$ & $280 / 299$ & 50.2 \\
$\left(t_{4}-t_{0}\right)$ & $305 \pm 2$ & $362 / 349$ & 29.0 \\
$\left(t_{5}-t_{0}\right)$ & $305 \pm 2$ & $370 / 399$ & \\
\hline
\end{tabular}
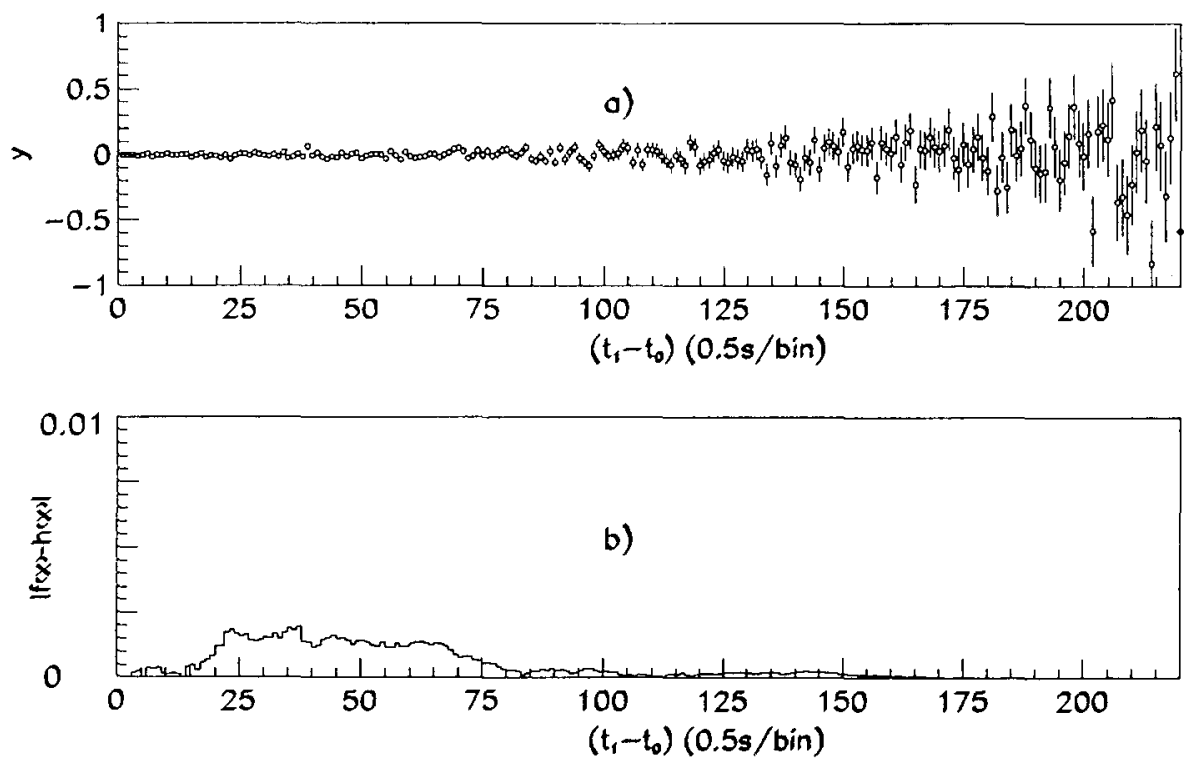

Fig. 6. (a) The quantity of fig. 4 vs. $\left(t_{1}-t_{0}\right)$ for the single muon data of period (D). (b) Results of the Kolmogorov-Smirnov test of the $\left(t_{1}-t_{0}\right)$ distribution for the same period (D) (see text). The probability of the random distribution is $P_{k}=88.4 \%$. 
The time region between 0 and 60 seconds was further explored in detail in both single and multiple muon samples: they do not show any indication of structure.

Fig. 4 shows the distribution $\left(t_{2}-t_{0}\right)$ of the following consecutive muons, for single muon events for data from period (D). The data have a smooth appearance, with no indications of structures, as confirmed by the plots of figs. $4 \mathrm{~b}, \mathrm{c}$.

The Kolmogorov-Smirnov test for the $\left(t_{\mathrm{M}}-t_{0}\right)$ distributions $(M=1 \div 5)$ gives probabilities $P_{k}$ greater than $14 \%$ for all distributions, thus confirming the agreement with the random distribution.

Fig. 5 shows the $\left(t_{3}-t_{0}\right),\left(t_{4}-t_{0}\right)$ and $\left(t_{5}-t_{0}\right)$ distributions for multimuon events of period (D). No deviations from smooth curves are seen.

\section{Time correlations with scintillators data}

In order to explore smaller time intervals we used the data from the scintillators from the first supermodule analyzed by the PHRASE circuits [11], which represent the dedicated circuitry for searches for neutrinos from stellar collapses.

The data for period (D) were selected using the same general criteria as for the streamer tube data, that is eliminating calibrations and runs with durations smaller than 3 hours. The dead time of this apparatus is about $3 \mathrm{~ms}$ for a single scintillation counter, but since it is unlikely that the next muon falls on the same scintillator, the effective dead time is at least 10 time smaller. Thus one can explore very small time intervals with almost no dead time losses.

The scintillation counters are grouped in three blocks: horizontal top layer, horizontal bottom layer and verticals. A muon is defined as a coincidence within 160 ns of two layers of scintillation counters. No other software cut is applied.

Fig. 7 shows the $\left(t_{1}-t_{0}\right)$ time distribution for single muons from period (D) analysed by the scintillators of the first supermodule. No structure is seen in the time interval $20 \mathrm{~ms}-10 \mathrm{~s}$. Fits of the distributions measured with the scintillators yield $\chi^{2} /$ d.o.f. $\sim 0.9$. The $\lambda$-value obtained from the scintillators may be compared with that obtained from the streamer tubes keeping in mind that the difference in effective surface is known to about $2 \%$. The $\lambda$-values agree to within this precision.

\section{Search for time correlations on a submicrosecond time scale}

MACRO is instrumented to measure time differences with 2 nanosecond resolution between muon induced signals up to about $180 \mathrm{~ns}$. We have used this to search for time correlations between muons in a single multimuon event in the interval of $18 \mathrm{~ns}$ to $180 \mathrm{~ns}$ after the average arrival time of a muon group. 


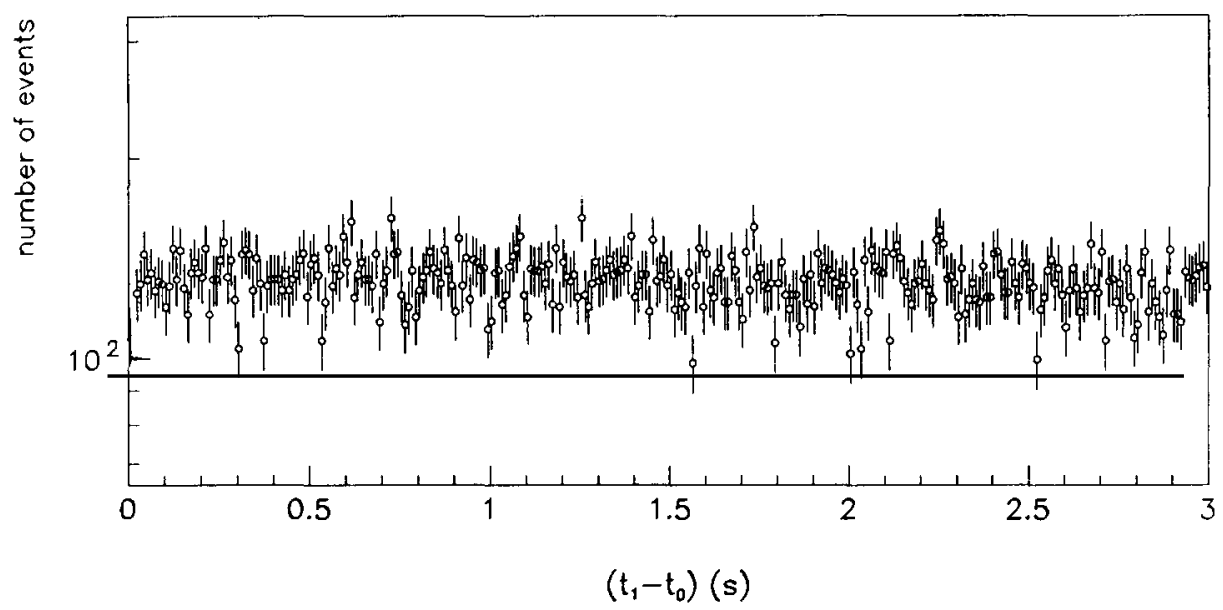

Fig. 7. The $\left(t_{1}-t_{0}\right)$ time distribution at small time intervals for the data of period (D) obtained by the scintillation counters (see text). Each bin is $10 \mathrm{~ms}$ wide.

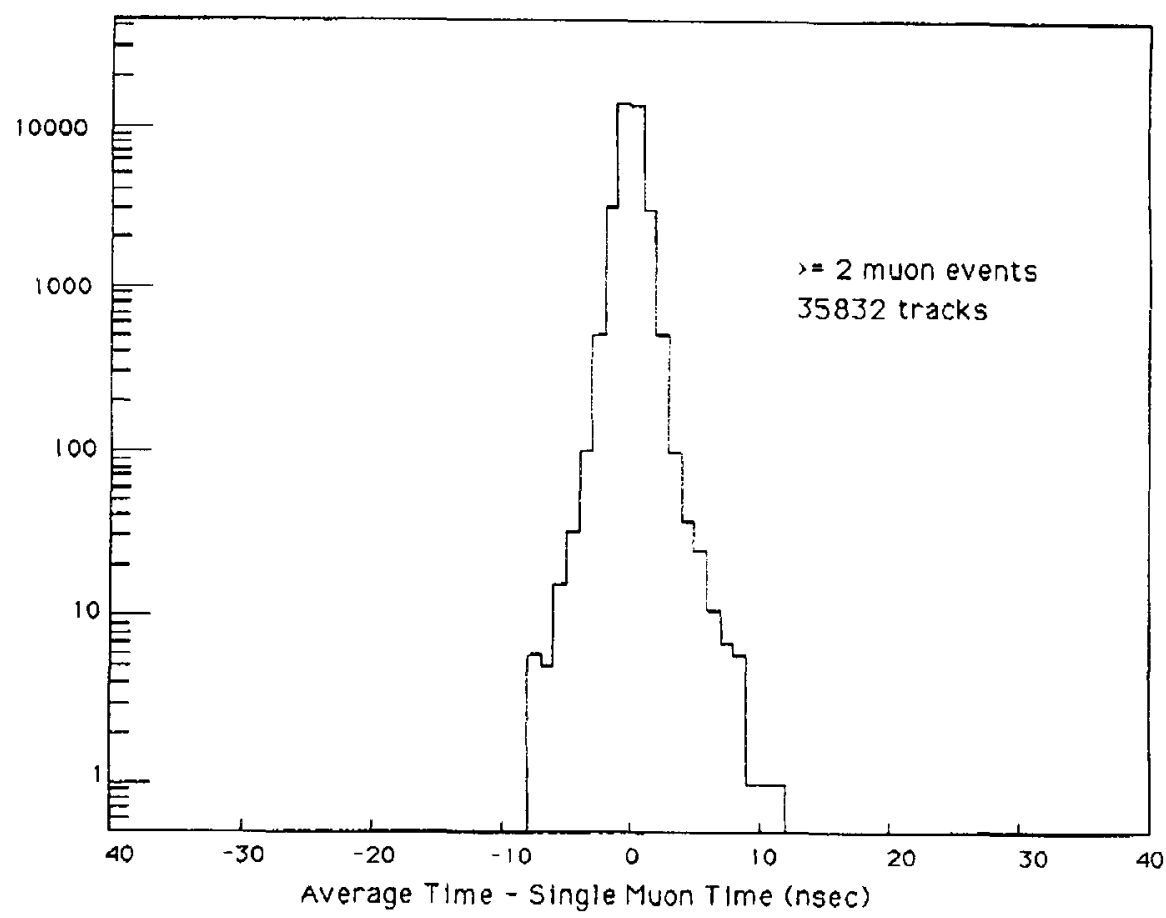

Fig. 8. Distributions of the time differences between the average arrival time and the arrival time of each muon for a sample of multi-muon events (notice the logarithmic scale in the vertical axis). There are no events in the range $18-180 \mathrm{~ns}$. 
The temporal decoherence between the several muons in a multi-muon event is a distribution covering about \pm 10 ns from the mean arrival time and this is shown in fig. 8 for 35832 tracks of events with two or more tracks (each having two counters intercepted and more than $4 \mathrm{~m}$ of path length). Here we are not addressing this decoherence distribution and we show it for reference only; it will be fully discussed in a future paper. We have observed no events in the 18-180 ns time interval. This is consistent with the number of events expected $(0.0022)$ from the number of single muon tracks with two counters intercepted (631735) and from the rate (about $0.021 \mathrm{~Hz}$ ).

\section{Conclusions}

We have presented data on the time of arrival distributions of cosmic ray muons with energies larger than $1.4 \mathrm{TeV}$ (at sea level) in the first two supermodules of the MACRO detector at the underground Gran Sasso National Laboratory. About $10^{6}$ selected events were used: the detailed analysis of period (D) (from June 21 till September 27,1990 ) involves 407420 muons. The muon arrival times, from milliseconds to several hundreds of seconds, follow closely the random distribution; there are no indications of deviations or of time anisotropies.

The search for nanosecond correlations in muons from the same muon bundle yields result consistent with the experimental time resolution, with no indication of delayed particles for times between 18 and $180 \mathrm{~ns}$.

We thank many colleagues for comments and discussions. We gratefully acknowledge the technical support provided by the Gran Sasso National Laboratory and by our Home Institutions. This work was supported by the Department of Energy and the National Science Foundation. L.M. and R.C. thank INFN for fellowships and financial support.

\section{References}

[1] T.C. Weekes, Nature 233 (1971) 129

[2] C.L. Bhat et al., Nature 288 (1980) 146

[3] G. Badino et al., Lett. Nuovo Cimento 28 (1980) 93

[4] D.J. Fegan et al., Proc. 17th ICRC, Paris (1981) Vol. 6, p. 296

[5] S. Higashi et al., Proc. 16th ICRC, Kyoto (1979), Vol. 12, p. 124

[6] V.D. Ashitkov et al., Proc. 17th ICRC, Paris (1981), Vol. 9, p. 344

[7] V.D. Ashitkov et al., Proc. 18th ICRC, Bangalore (1983), Vol. 7, p. 129

[8] C. Morello et al., Nuovo Cimento 7C (1984) 682

[9] P. Serri (Studio dei muoni penetranti nell'esperimento NUSEX del Monte Bianco), Tesi di Laurea, Università di Milano (1984)

[10] L. Sun, Proc. 19th ICRC, San Diego, California (1985)

[11] Macro Collaboration, S.P. Ahlen et al., Phys. Lett. B249 (1990) 149

[12] J.W. Elbert et al., Phys. Rev. D12 (1975) 660

[13] W.T. Eadie et al., Statistical methods in experimental physics (North-Holland, Amsterdam, 1971) p. 269 